\title{
PHENIX global observables and fluctuations from the RHIC beam energy scan
}

\author{
J.T. Mitchell for the PHENIX Collaboration* \\ Brookhaven National Laboratory \\ E-mail: mitchell@bnl.gov
}

The PHENIX Experiment at RHIC has participated in a beam energy scan at several collision energies in order to probe various regions of the QCD phase diagram in a seach for signatures of the QCD critical point and the onset of deconfinement. Measurements of transverse energy production at mid-rapidity will be discussed and examined to estimate the Bjorken energy density and quark participant scaling features. Also discussed are measurements of net charge fluctuation results.

9th International Workshop on Critical Point and Onset of Deconfinement - CPOD2014,

17-21 November 2014

ZiF (Center of Interdisciplinary Research), University of Bielefeld, Germany

* Speaker. 


\section{Introduction}

The PHENIX Experiment at Brookhaven National Laboratory's Relativistic Heavy Ion Collider (RHIC) has been participating in a beam energy scan program whose goal is to probe different regions of the QCD phase diagram in a search for the critical point predicted by QCD calculations. The program has been taking data from 2000-2014 with $\mathrm{Au}+\mathrm{Au}$ datasets taken at the following energies: $200,62.4,39,27,19.6,14.5$, and $7.7 \mathrm{GeV}$. An additional dataset at $130 \mathrm{GeV}$ was collected in 2001. This report shows the first results from the $14.5 \mathrm{GeV} \mathrm{Au}+\mathrm{Au}$ dataset, which was taken in 2014.

\section{Transverse Energy Production and Bjorken Energy Density}

Figure 1 shows the value of $d E_{T} / d \eta$ normalized by the number of nucleon participant pairs, $N_{\text {part }}$, for central collisions ( $0-5 \%$ centrality for PHENIX data) as a function of $\sqrt{s_{N N}}$. The data below $100 \mathrm{GeV}$ follow a linear trend as indicated by the line, which is a linear fit to all of the data points excluding the LHC data point. The LHC data point exceeds this trend by more than a factor of two.

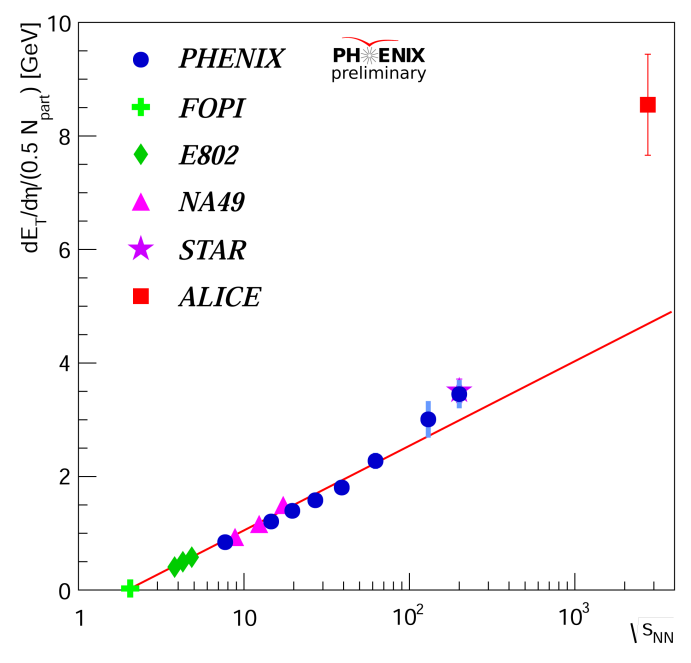

Figure 1: The value of $d E_{T} / d \eta$ at mid-rapidity normalized by the number of nucleon participant pairs as a function of $\sqrt{s_{N N}}$ for $\mathrm{Au}+\mathrm{Au}$ collisions. The red line is an exponential fit to all of the data points excluding the ALICE point. The ALICE data are taken from [1].

The trend followed by transverse energy production can be examined in terms of the Bjorken energy density [2], which is given by

$$
\varepsilon_{B j}=\frac{1}{A_{\perp} \tau} \frac{d E_{T}}{d y} .
$$

Figure 2 shows the Bjorken energy density multiplied by $\tau$ as a function of $\sqrt{s_{N N}}$ on a $\log -\log$ plot for the most central collisions. The straight line shows that a power law fit to all of the data points describes the data well with a power of 0.41 . 


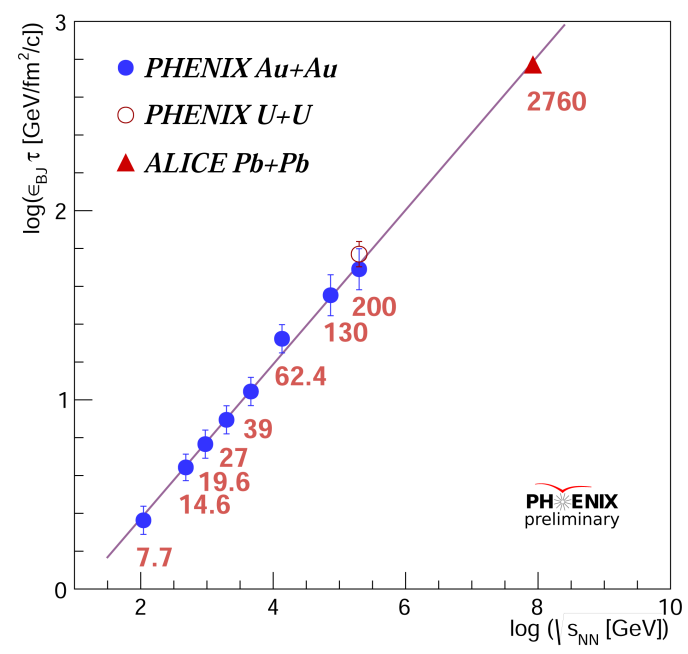

Figure 2: The log of the Bjorken energy density multiplied by $\tau$ for central collisions collisions versus the $\log$ of $\sqrt{s_{N N}}$. The purple line is a power law fit to all of the data points. The numbers state the value of $\sqrt{s_{N N}}$ for each point.

\section{Nucleon Participant and Quark Participant Scaling}

It has been well established that charged particle multiplity and transverse energy production at the top RHIC energies does not scale well with the number of nucleon participant pairs [3, 4]. Now, with the availability of the RHIC beam energy scan data, it is possible to determine if this scaling holds for lower energy collisions and begin to isolate the collision energy at which the scaling begins to break down. Since the overall behavior of both of these observables $\left(d N_{c h} / d \eta\right.$ and $\left.d E_{T} / d \eta\right)$ is identical, only transverse energy production will be discussed in this section. Figure 3 shows $d E_{T} / d \eta$ normalized by the number of nucleon participant pairs as a function of the number of nucleon participants. This figure shows that as the collision energy decreases, especially below $62 \mathrm{GeV}$, the data begin to scale well with the number of nucleon participants. This figure includes the $14.5 \mathrm{GeV} \mathrm{Au+Au}$ data taken this year. In order to illustrate this, a variable $R_{P C}$ is constructed as follows:

$$
R_{P C}=\frac{d E_{T} / d \eta}{d E_{T} / d \eta[0-5 \% \text { centrality }]}
$$

If the data scales with the number of nucleon participants, then the data should lie on the $R_{P C}=$ 1 line. Figure 4 shows the value of $R_{P C}$ for all of the datasets from the RHIC beam energy scan. The data below $62 \mathrm{GeV}$ are consistent with $N_{\text {part }}$ scaling. Note that the LHC data do not scale with $N_{\text {part }}$.

There have been previous studies of the scaling of particle production with the number of quark participants [5, 6]. In this study, a Glauber model calculation [7] is used to estimate the number of quark participants as a function of the centrality of the collision. For this estimate, nucleons are first distributed within each nucleus according to a Woods-Saxon distribution. Each nucleon is then replaced by three quarks distributed around the center of each nucleon with a 


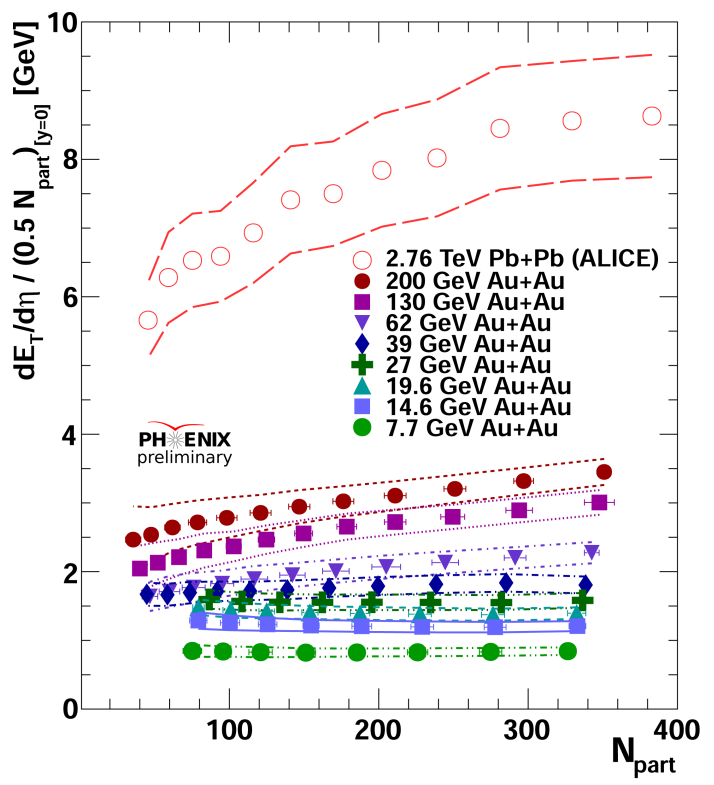

Figure 3: The value of $d E_{T} / d \eta$ normalized by the number of nucleon participant pairs at mid-rapidity as a function of $N_{\text {part }}$. Error bars represent statistical errors and the lines define the boundaries of the systematic errors for each system.

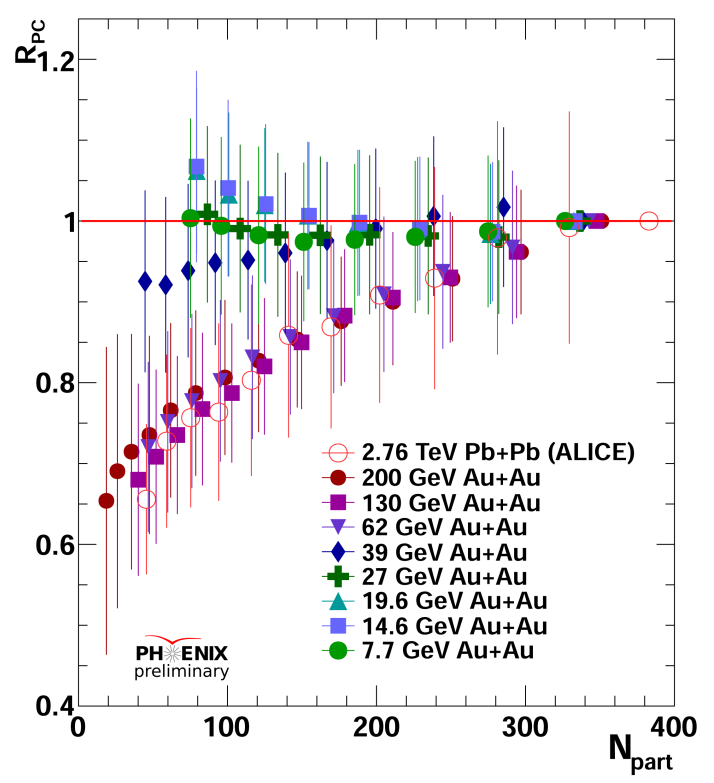

Figure 4: The value of $R_{P C}$ plotted as a function of $N_{\text {part }}$. The red line is a constant line at $R_{P C}=1$. The error bars represent systematic errors, which can be correlated from point to point. 
radial distribution sampled from the proton form factor. A pair of quarks from each nucleus are considered to interact with each other if their separation, $d$, in the plane transverse to the beam axis satisfies the condition $d<\sqrt{\sigma_{q q}^{\text {inel }} / \pi}$, where $\sigma_{q q}^{\text {inel }}$ is the inelastic quark-quark cross section. The value of $\sigma_{q q}^{\text {inel }}$ is determined by varying its value for the case of nucleon-nucleon collisions until the known inelastic nucleon-nucleon cross section is reproduced. The number of quark participants, $N_{\text {quark-part }}$, as a function of centrality is determined using the procedure described in [8]. More details on the simulation can be found in [9].

In Figure 5, $d E_{T} / d \eta$ scaled by the number of quark participant pairs is plotted as a function of $N_{\text {quark-part }}$ for all of the beam energy scan data sets. The plot shows that the higher energy datasets scale better with $N_{\text {quark-part }}$ than the lower energy datasets, especially above $39 \mathrm{GeV}$. Figure 6 shows the data displayed in terms of the $R_{P C}$ variable, illustrating that the RHIC data above 39 $\mathrm{GeV}$ are consistent with $N_{\text {quark-part }}$ scaling while the lower energy data are not. It is interesting that the LHC data are less consistent with $N_{\text {quark-part }}$ scaling than the $200 \mathrm{GeV} \mathrm{Au}+\mathrm{Au}$ data.

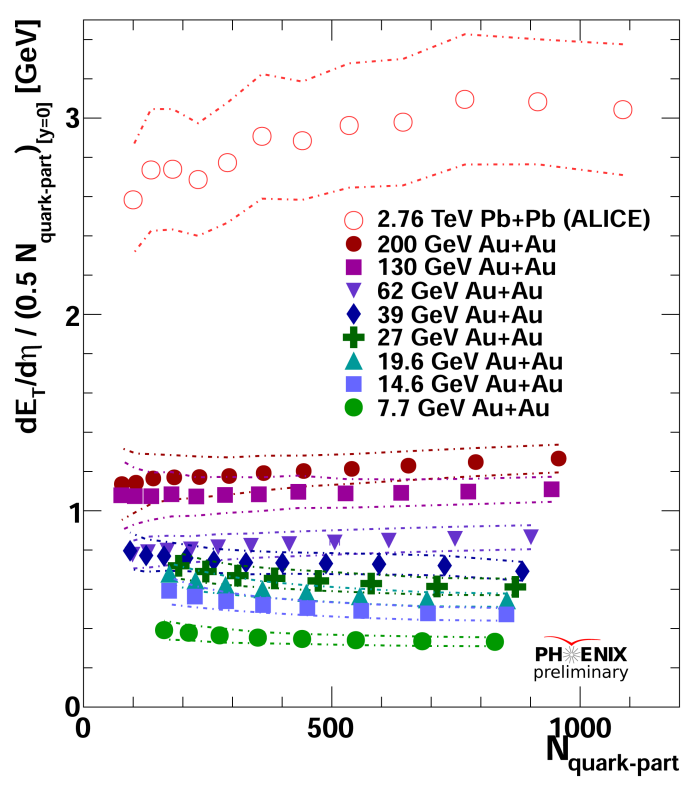

Figure 5: The value of $d E_{T} / d \eta$ normalized by the number of quark participant pairs at mid-rapidity as a function of $N_{\text {quark-part }}$. Error bars represent statistical errors and the lines define the boundaries of the systematic errors for each system.

It is possible to estimate the transverse energy production per quark participant at mid-rapidity. In order to obtain this estimate, $d E_{T} / d \eta$ is plotted as a function of $N_{\text {quark-part }}$ and then fit to a straight line over a region where there is a good linear fit for all energies $\left(N_{\text {quark-part }}>400\right)$. The slopes of the fits are plotted in Figure 7 as a function of the $\log$ of $\sqrt{s_{N N}}$. The data are well described by a second-order polynomial function with coefficients $p_{0}=0.139, p_{1}=-0.0475$, and $p_{2}=0.0282$. 


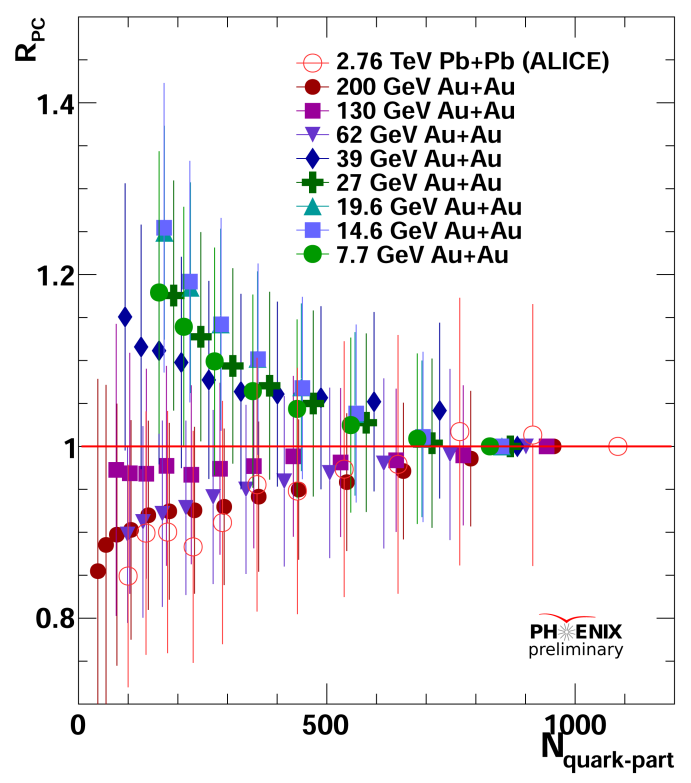

Figure 6: The value of $R_{P C}$ plotted as a function of $N_{\text {quark-part }}$. The red line is a constant line at $R_{P C}=1$. The error bars represent systematic errors, which can be correlated from point to point.

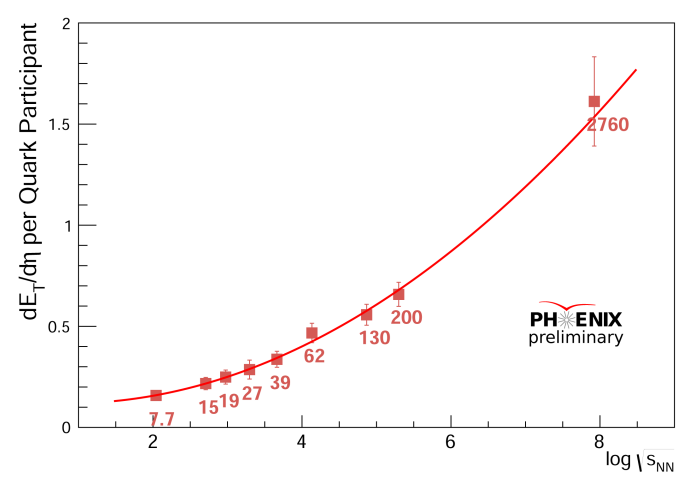

Figure 7: The $d E_{T} / d \eta$ produced per quark participant at mid-rapidity in central collisions as a function of the $\log$ of $\sqrt{s_{N N}}$. The line is a second-order polynomial fit to the data.

\section{Net Charge Fluctuations}

PHENIX has also searched for direct evidence of the existence of the QCD critical point by measuring the fluctuations of the net charge on an event-by-event basis. The shapes of the distributions of the net charge should be sensitive to the presence of the critical point [10]. PHENIX has measured the skewness $\left(S=\left\langle(N-\langle N\rangle)^{3}\right\rangle / \sigma^{3}\right)$ and the kurtosis $\left(\kappa=\left\langle(N-\langle N\rangle)^{4}\right\rangle / \sigma^{4}-3\right)$ of net charge distributions in Au+Au collisions at $\sqrt{s_{N N}}=200,62.4,39$, and 7.7 GeV. The measurements are quoted in terms that can be associated with quark number susceptibilities, $\chi: S \sigma \approx \chi^{(3)} / \chi^{(2)}$ 
and $\kappa \sigma^{2} \approx \chi^{(4)} / \chi^{(2)}$ [11]. The skewness and kurtosis for central collisions at mid-rapidity are shown in Figure 8 as a function of $\sqrt{s_{N N}}$. The data are compared to URQMD and HIJING simulation results processed through the PHENIX acceptance and detector response. The data show that there is no significant excess above the simulation results observed at these four collision energies.

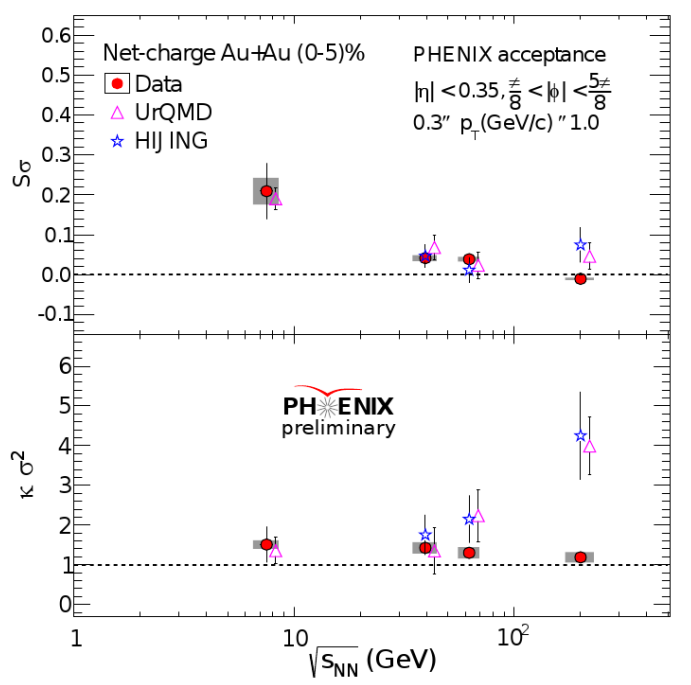

Figure 8: The skewness multiplied by the standard deviation and the kurtosis multiplied by the variance from net charge distributions in central $\mathrm{Au}+\mathrm{Au}$ collisions. The circles represent the data. The grey error bars represent the systematic errors. Also shown are URQMD and HIJING simulation results processed through the PHENIX acceptance. The increase in the kurtosis from URQMD and HIJING may be due to an increase in resonance production at $200 \mathrm{GeV}$.

\section{5. sPHENIX Beam Energy Scan II Plans}

The RHIC Beam Energy Scan II program is currently planned to span two years of RHIC running from 2018 to 2019. The program will utilize a bunched beam electron cooling upgrade of RHIC to improve the operating luminosity at low energies. The PHENIX plans for participation in the RHIC Beam Energy Scan II program (BES-II) is detailed in a white paper [12], which will be summarized in this section. PHENIX is currently preparing for its final year of running using the current detector configuration in 2016. After the 2016 running period, the PHENIX detector will be disassembled and the sPHENIX detector will be installed. The sPHENIX detector features full azimuthal coverage within $|\eta|<1.1$ and includes hermetic calorimetry and tracking over the entire acceptance. However, installation of the sPHENIX detector cannot be completed in time during the planned 18-20 month gap between the 2016 run and the BES-II program. Therefore, sPHENIX will only be able to participate starting with the second year of the BES-II program. The sPHENIX detector [13] for the 2019 running period will consist of the solenoidal magnet previously used by BABAR, the hadronic calorimeter, any available portion of the electromagnetic calorimeter, and either a reconfigured PHENIX silicon vertex detector or possibly a Time Projection Chamber. A 
schematic diagram of the sPHENIX detector configuration with the silicon vertex detector is shown in Figure 9.

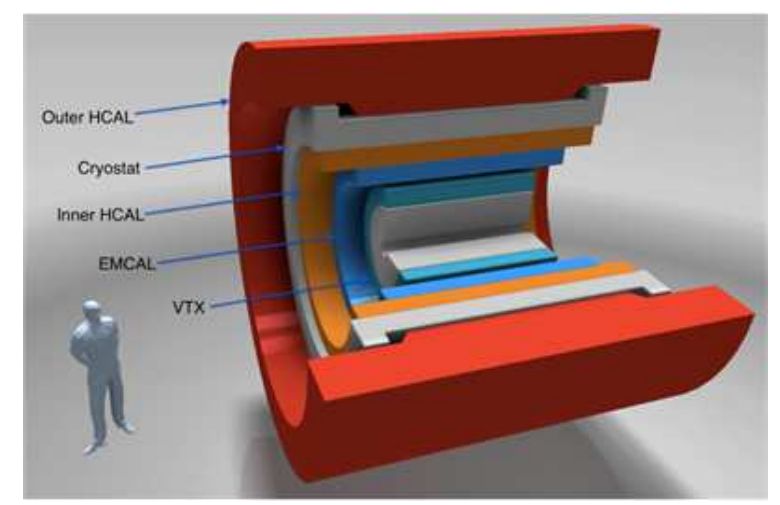

Figure 9: A schematic diagram of the sPHENIX detector configuration for the BES-II program.

sPHENIX is interested in running at BES-II energies where PHENIX has not yet taken data. PHENIX did not take data at $11.5 \mathrm{GeV}$ during the BES-I program due to technical constraints of the collider at the time, so sPHENIX is interested in taking data at the intriguing energies of 9.0, 11.5, and $13.0 \mathrm{GeV}$. sPHENIX is also requesting to take $200 \mathrm{GeV} \mathrm{Au+Au}$ data in order to assist in the commissioning of the sPHENIX detector for its primary physics program. With the statistics expected for one year of BES-II running with sPHENIX and using the net proton kurtosis errors following the formulae outlined in [15], an estimate of the expected errors on a net proton kurtosis measurement is shown in Figure 10. sPHENIX should be able to significantly improve on the net proton kurtosis errors published by the STAR collaboration from BES-I [14].

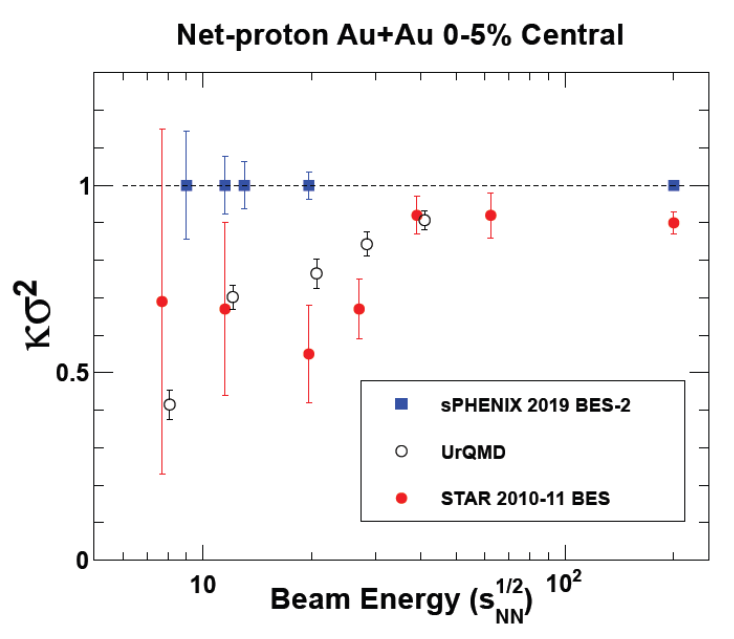

Figure 10: The estimated errors for net proton kurtosis expected for sPHENIX for various collision energies from the Beam Energy Scan II program. The errors are estimated using the prescription outlined in [15]. For comparison, the errors obtained by the STAR collaboration from the BES-I program are shown. Also shown are estimates of the errors from URQMD simulations. 


\section{Summary}

The PHENIX Experiment has performed a study of transverse energy production at several collision energies. The Bjorken energy density exhibits power law scaling as a function of the logarithm of $\sqrt{s_{N N}}$ from $7.7 \mathrm{GeV}$ to $2760 \mathrm{GeV}$. It is observed that $d E_{T} / d \eta$ normalized by the number of participant pairs scales well with the number of nucleon participants below $39 \mathrm{GeV}$, and shows better scaling with the number of quark participants above $39 \mathrm{GeV}$. Measurements of higher moments of the net charge distribution do not show any significant excess that would be indicative of the presence of a critical point. Currently, PHENIX is planning to participate in the second half of the Beam Energy Scan II program using the sPHENIX detector upgrade.

\section{References}

[1] C. Loizides et al., arXiv:1106.6324v1 (2011).

[2] J. D. Bjorken, Phys. Rev. D 27, 140 (1983).

[3] B. Alver et al., Phys. Rev. C 83, 024913 (2011).

[4] S.S. Adler et al., Phys. Rev. C 71, 034908 (2005).

[5] S. Eremin and S. Voloshin, Phys. Rev. C 67, 064905 (2003).

[6] R. Nouicer, Eur. Phys. J. C 49, 281 (2007).

[7] B. Alver et al., arXiv:0805.4411 (2008).

[8] M.L. Miller et al., Annu. Rev. Nucl. Part. Sci. 2007. 57:205-43 (2007).

[9] S.S. Adler et al., Phys. Rev. C 89, 044905 (2014).

[10] R. V. Gavai and S. Gupta, Phys. Lett. B 696, 459 (2011).

[11] F. Karsch and K. Redlich, Phys. Lett. B 695, 136 (2011).

[12] Available for download at http://www.phenix.bnl.gov/plans.html.

[13] Available for download at http://www.phenix.bnl.gov/plans.html.

[14] L. Adamczyk et al., Phys. Rev. Lett. 112, 032302 (2014).

[15] X. Luo, J. Phys. G: Nucl. Part. Phys. 39, 025008 (2012). 